\title{
Particle Acceleration in Gamma-Ray Bursts
}

\author{
J.G. Kirk \\ Max-Planck-Institut für Kernphysik, Postfach 1039 80, 69029 Heidelberg, \\ Germany; \\ john.kirk@mpi-hd.mpg.de
}

\begin{abstract}
Summary. Simple kinematic theories of particle acceleration at relativistic shocks lead to the prediction of a high-energy spectral index of -1.1 for the energy flux of synchrotron photons. However, several effects can change this picture. In this paper I discuss the effect of magnetic field generation at the shock front and, by analogy with the Crab Nebula, suggest that an intrinsic break in the injection spectrum should be expected where the electron gyro radius is comparable to that of protons thermalized by the shock.
\end{abstract}

\section{Introduction}

The process or processes which accelerate the particles responsible for the nonthermal emission observed in gamma-ray bursts and in their after-glows are still unknown. However, the only proposed mechanism with significant predictive power is the first-order Fermi process operating at a shock front. In nonrelativistic flows, this mechanism has been applied in a wide variety of astrophysical situations and has been the subject of considerable theoretical effort - with interest currently focused on nonlinear aspects [16]. The relativistic outflow from a gamma-ray burst, on the other hand, demands a different approach [11], which is able to account for the intrinsic anisotropy of the particle distribution functions. In this paper I briefly review the status of the relativistic theory, present some new calculations of test-particle acceleration in the presence of shock-generated magnetic field, and discuss the application of the process to the modeling of GRB after-glow spectra, stressing an analogy with models of the relativistic termination shock of the wind from the Crab pulsar.

\section{Kinematics}

The kinematic problem of particle acceleration at a relativistic shock, i.e., that of finding the distribution of a collection of test particles undergoing small-angle, random, elastic (in the plasma frame) deflections in the vicinity of a discontinuity in the (relativistic) plasma velocity is well-understood. An analytic method based on an eigenvalue decomposition is available which 

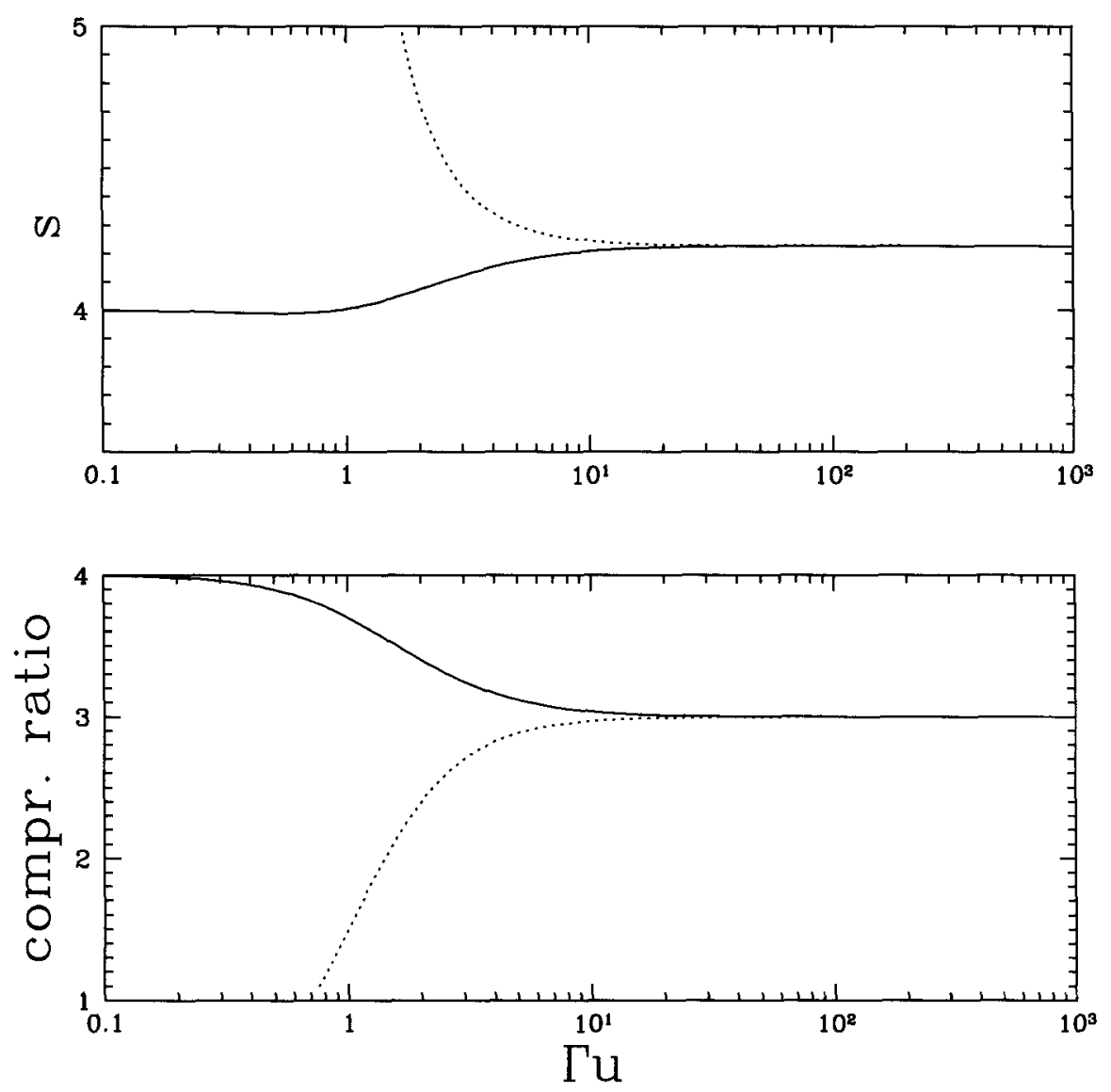

Fig. 1. The high-energy power-law index $s$ (upper panel) and compression ratio (lower panel) as a function of the spatial component of the upstream four speed $\Gamma u$. The dotted line refers to a shock in a gas with negligible rest-mass and the solid line to a strong shock (i.e., cold upstream medium) in an ideal gas with adiabatic. index $5 / 3$.

gives the spectrum and angular dependence of the distribution function at energies well above those of injection for arbitrary shock speeds [12]. In addition, Monte-Carlo simulations have been performed, finding results which are in good agreement with the analytic approach $[1,3]$. These results are illustrated in Figs. 1 and 2. Well above the injection energy the phase-space density $f$ is a power-law in momentum: $f \propto p^{-s}$ and at the shock front the angular dependence is well-approximated by the simple expression

$$
f \propto\left(1-\mu_{\mathrm{s}} u\right)^{-s} \exp \left(-\frac{1+\mu_{\mathrm{s}}}{1-\mu_{\mathrm{s}} u}\right)
$$


downstream flux distribution

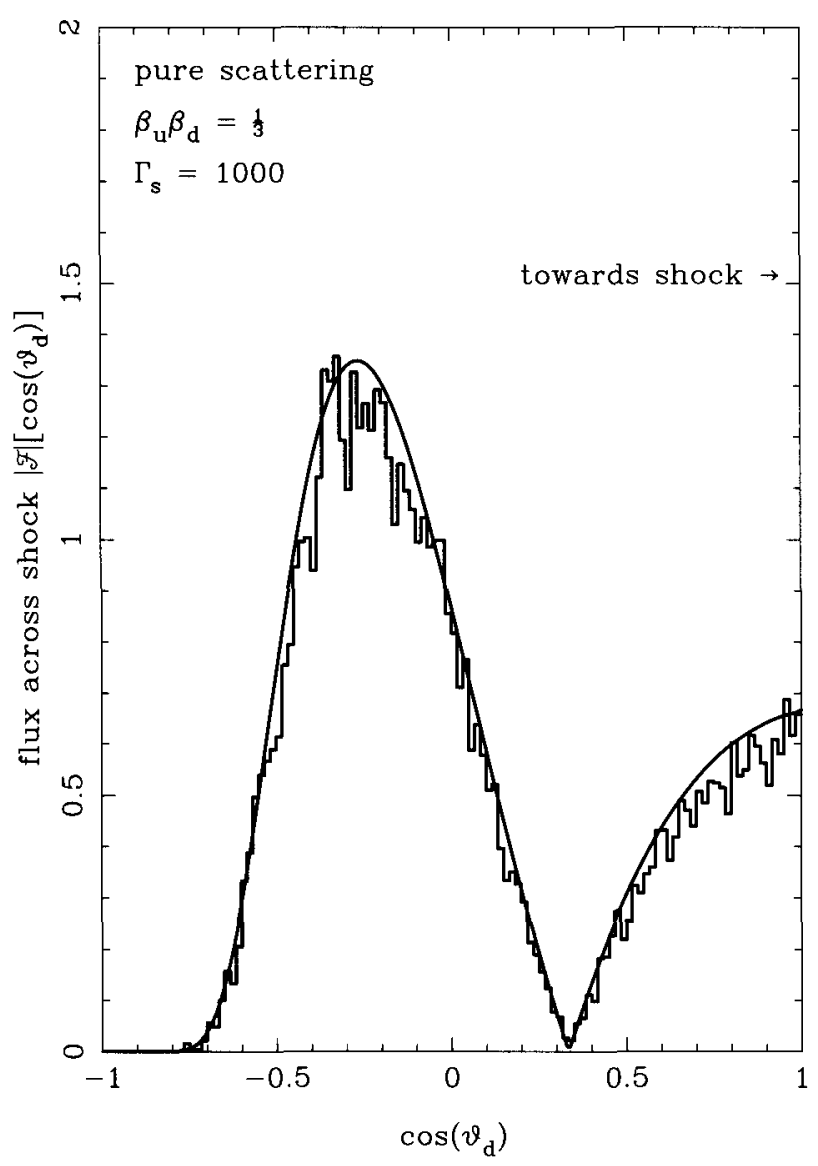

Fig. 2. A comparison (from [1]) between a Monte-Carlo simulation and the analytic result for the particle flux across a shock front as a function of the cosine of the angle $\theta_{\mathrm{d}}$ between the particle velocity and the shock normal measured in the frame in which the downstream plasma is at rest. $\theta_{\mathrm{d}}=0$ corresponds to motion along the normal from downstream to upstream. Jump conditions for a relativistic gas are used and the upstream plasma has a Lorentz factor $\Gamma=1000$.

where $\mu_{\mathrm{s}}$ is the cosine of the angle between the shock normal and the particle velocity $c u$ measured in the frame in which the shock is at rest and the upstream plasma flows along the shock normal. Fig. 1 shows the compression ratio and the high-energy power-law index $s$ as a function of the spatial component of the 4-speed $\Gamma u$ of the upstream plasma, where $\Gamma=\left(1-u^{2}\right)^{-1 / 2}$.

For gamma-ray bursts, an interesting aspect of these results is that the power-law index tends asymptotically to the value $s \approx 4.23$ for large shock Lorentz factors (or, equivalently, upstream Lorentz factors), independent of 
the equation of state of the plasma. This asymptotic value is essentially fixed by the compression ratio of the shock and depends only weakly on the form of the scattering operator used to describe the small-angle deflections [12].

There is an important difference between the scattering operator conventionally used in nonrelativistic theory and that used for relativistic shocks. The nonrelativistic picture assumes it is reasonable to define the trajectory of a particle between scatterings (the unperturbed motion) in terms of the motion of its guiding center. Scatterings cause a change in the pitch-angle, leading to the diffusion of particles along magnetic field lines. Cross-field diffusion is suppressed in this picture. However, as seen in the frame of the downstream medium, the magnetic field carried towards a shock by an upstream plasma flowing at high Lorentz factor appears to lie almost in the plane of the shock front. As a consequence, relativistic shocks are perpendicular shocks [4] which cannot be multiply crossed by particles diffusing along field lines. The first-order Fermi mechanism does not operate in this configuration unless cross-field diffusion is effective.

In a uniform field, a particle which crosses a relativistic shock front from downstream to upstream will be recaptured by the front after executing a fraction of roughly $\sim 1 / \Gamma$ of a gyration. Thus, if scattering plays a role, it is reasonable to describe the unperturbed trajectory not as a helix, but as a straight line. This is especially true if, as expected (see next section), the field is highly nonuniform on the length seale of a gyro radius. In this case the role of the average field (if it exists) ceases to be important, and the description of the stochastic trajectory is in terms of deflections of the velocity, rather than changes in pitch angle. This is the form of operator used in the analytic approach. In Monte-Carlo treatments, it is possible to retain the effect of an average field $[1,18]$. Provided the turbulence remains strong, little difference is found. However, as expected, the acceleration mechanism becomes less effective as the turbulence diminishes [19]. Explicit calculations of particle motion in a random magnetic field have also been performed $[2,5]$ and used to compute the acceleration around a relativistic shock for Lorentz factors $\Gamma \leq 5[2]$ and, more recently, for $\Gamma \leq 100$ [14]. The latter find good agreement with the analytic result on the asymptotic power-law index.

\section{Nonlinear Effects and Magnetic Field Generation}

In contrast with the situation in nonrelativistic shocks [16], the nonlinear modification of relativistic shock does not affect the asymptotic power-law index. There are two reasons for this: firstly, isotropized, accelerated particles behave like a relativistic gas with adiabatic index $4 / 3$, so that the overall compression ratio of an ultra-relativistic shock front remains 3 , even when a significant part of the overall energy and momentum flux is carried by these particles. Secondly, the asymptotic power-law index in the test-particle picture is soft (i.e., $s>4$ ). This means that it is possible to consider a 
Lorentz factor above which the test-particle approximation is valid, because the energy density in the remaining accelerated particles is indeed small. Nevertheless, a strong nonlinear effect can be exerted by particles of lower energies, whose mean free path to scattering is comparable to the size of internal structures in the shock transition [7].

Although there are strong indications that collisional processes may be important in the inner parts of a GRB fireball $r<10^{16} \mathrm{~cm}[6,23]$, the plasma responsible for the after-glow is probably best treated as collisionless. The most promising way of forming a relativistic shock in such a plasma is by the Weibel instability $[17,25,26]$, which generates downstream magnetic field perpendicular to the streaming motion of the plasma i.e., in the plane of the incipient shock. A full simulation of this situation has not yet been performed, but recent 3 D-PIC simulations of colliding plasma shells $[8,21,22]$ suggest that magnetic field can be generated with a strength up to $\sigma \approx 1 \%$. (Here the magnetization parameter $\sigma$ is defined as the ratio of the magnetic energy density to twice the total enthalpy density (including rest mass) as measured in the plasma rest frame). This is encouraging, since it is roughly the level implied by spectral modeling [20] of GRB after-glows.

The spectrum of accelerated particles is certainly closely tied to the evolution of the turbulent magnetic field. However, if we are interested only in high energy particles of long mean free path, the power-law index predicted by the first-order Fermi mechanism can be calculated simply by modifying the shock jump conditions to account for the generated field. To do this, we consider time-averaged conditions, so that linear functions of the electromagnetic field vanish. The stress-energy tensor in the plasma frame is

$$
T^{\mu \nu}=\left(w+\frac{B^{2}}{4 \pi}\right) u^{\mu} u^{\nu}+\left(p+\frac{B^{2}}{8 \pi}\right) g^{\mu \nu}-\frac{B^{\mu} B^{\nu}}{4 \pi}
$$

(for notation see [11]) and the last term on the right hand side does not contribute to the fluxes across the shock front if the magnetic field lies in the shock plane. As a result, the jump conditions are the same as those of an unmagnetized fluid, provided the magnetic enthalpy density $B^{2} / 4 \pi$ and pressure $B^{2} / 8 \pi$ are taken into account [15]. For a relativistic gas, this gives an effective adiabatic index

$$
\gamma_{\mathrm{eff}}=\frac{4(1+\sigma)}{(3+\sigma)}
$$

leading to an asymptotic compression ratio of $1 /\left(\gamma_{\text {eff }}-1\right)$ and a relative speed of the upstream medium with respect to the downstream medium corresponding to the Lorentz factor $\Gamma_{\text {rel }}=\Gamma \sqrt{\left(2-\gamma_{\mathrm{eff}}\right) / \gamma_{\mathrm{eff}}}$ (where $\Gamma$ is the Lorentz factor of the shock front seen in the upstream medium). As $\sigma$ increases, the compression ratio of the shock decreases and the high-energy power-law softens, as shown in Fig. 3. 

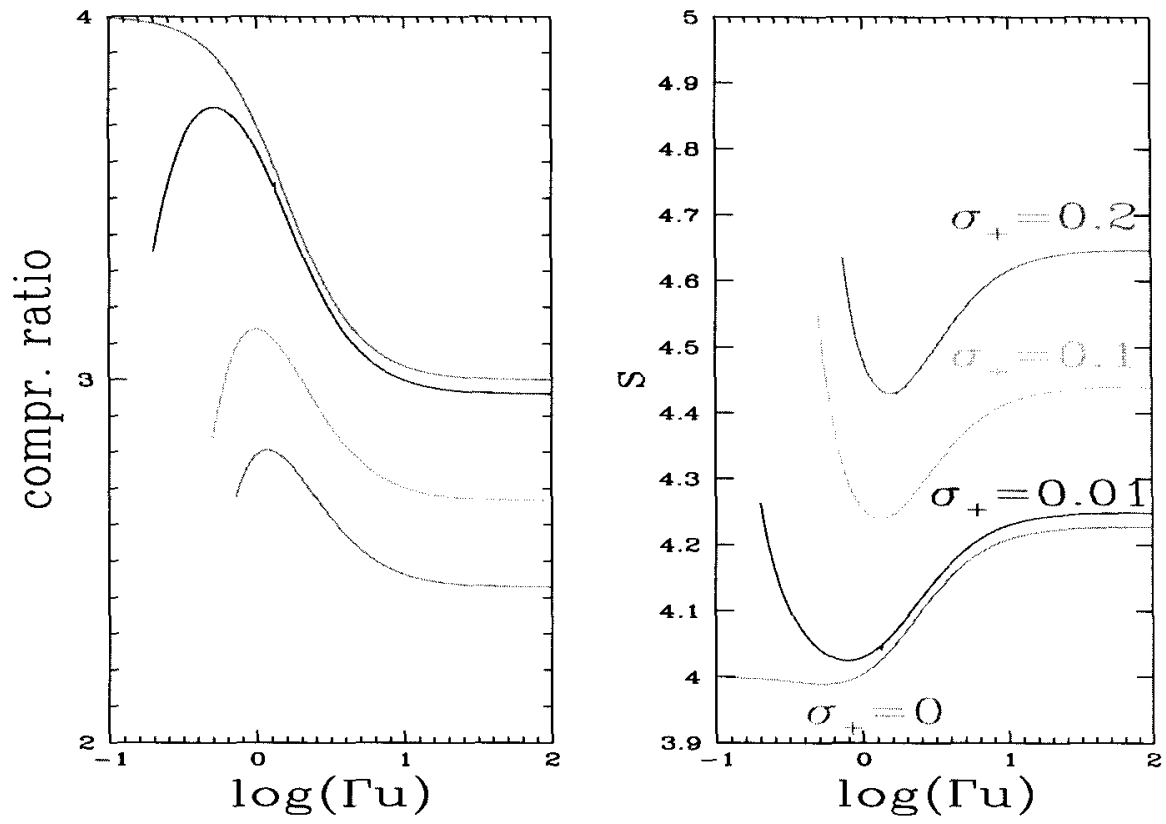

Fig. 3. The compression ratio and power-law index $s$ for a gas of adiabatic index $5 / 3$ in which a magnetic field is generated downstream to the level $\sigma=\sigma_{+}$(see text for notation).

\section{Learning from the Crab}

A relativistic wind carries energy from the Crab pulsar out to the Nebula. This is released into nonthermal particles at a termination shock, which is probably the best observed relativistic shock in the universe. The average Lorentz factor of the upstream plasma can be estimated from the spin-down luminosity of the pulsar and the total number of electrons and positrons which have accumulated in the Nebula [13] to be between $\sim 10^{4}$ and $10^{6}$. This is well into the asymptotic region of high $\Gamma$ as far as the first-order Fermi process is concerned, and the X-ray synchrotron emission [24] indeed corresponds to the predicted power-law index of $s=4.2$ for a plasma in which the magnetic energy plays no dynamical role [9].

However, the integrated synchrotron spectrum displays not only the expected "cooling break", at a frequency where the characteristic cooling time corresponds roughly to the age of the object, but also a second break at higher frequency [9]. This is presumably due to an intrinsic feature of the acceleration process and probably characterizes the transition between two different mechanisms. One possibility is that the break energy reflects the different spatial scales associated with ions and electrons/positrons. Regarded as distinct fluids, these components would be expected to produce shock structures 
each on the scale of the thermal gyro radius: $\Gamma M c^{2} / e B$ and $\Gamma m c^{2} / e B$, respectively. However, strong heating of the electron/positron gas, perhaps via the maser mechanism proposed by Hoshino et al. [10], should result in a smearing out of the smaller of these two scales. Below Lorentz factors of roughly $\Gamma M / m$, the hard $(s \approx 3)$ maser mechanism should dominate over the first-order mechanism for electrons and positrons. An alternative explanation, which assumes the ions in the Crab wind carry very little of the energy flux, is that magnetic field dissipation within the shock front is responsible $[15]$.

Whichever (if any) of these speculations is correct, the implications for GRB models are that the intrinsic spectrum is unlikely to be a single powerlaw. Hard spectra $(s<4)$ concentrate energy at the highest Lorentz factors and are not expected from the first-order Fermi mechanism at a relativistic shock. The signature of the latter is a softer spectrum extending above the bulk of the distribution with a power-law index which ranges from 4.2 to about 4.4 , depending on the efficiency of magnetic field generation.

\section{References}

1. A. Achterberg, Y.A. Gallant, J.G. Kirk, A.W. Guthmann: Mon. Not. R. Astron. Soc. 328, 393 (2001)

2. K.R. Ballard, A.F. Heavens: Mon. Not. R. Astron. Soc. 259, 89 (1992)

3. J. Bednarz, M. Ostrowski: Phys. Rev. Lett. 80, 3911 (1998)

4. M.C. Begelman, J.G. Kirk: Astrophys. J. 353, 66 (1990)

5. F. Casse, M. Lemoine, G. Pelletier: Phys. Rev. D 65, 023002 (2002)

6. E.V. Derishev, F.A. Aharonian, V.V. Kocharovsky, Vl.V. Kocharovsky: astroph 0301263 (2003)

7. D.C. Ellison, G.P. Double: Astropart. Phys. 18, 213 (2002)

8. R.A. Fonseca, L.O. Silva, J.W. Tonge, W.B. Mori, J.M. Dawson: Phys. Plasmas 10, 1979 (2003)

9. Y. Gallant, E. van der Swaluw, J.G. Kirk, A. Achterberg: "Modeling plerion spectra and their evolution." In: Neutron Stars in Supernova Remnants. eds. P.O. Slane, B.M. Gaensler (ASP Conf. Series: Vol. 271, 2001) p. 161

10. M. Hoshino, J. Arons, Y.A. Gallant, A.B. Langdon: Astrophys. J. 390, 454 (1992)

11. J.G. Kirk, P. Duffy: J. Phys. G: Nucl. Part. Phys. 25, R163 (1999)

12. J.G. Kirk, A.W. Guthmann, Y.A. Gallant, A. Achterberg: Astrophys. J. 542, $235(2000)$

13. J.G. Kirk, O. Skjæraasen: Astrophys. J. 591, 366 (2003)

14. M. Lemoine, G. Pelletier: Astrophys. J. 589, L73 (2003)

15. Y. Lyubarsky: astro-ph 0306435 (2003)

16. M. Malkov, L.O'C. Drury: Rep. Prog. Phys. 64, 429 (2001)

17. M.V. Medvedev, A. Loeb: Astrophys. J. 526, 679 (1999)

18. M. Ostrowski: Mon. Not. R. Astron. Soc. 264, 248 (1993)

19. M. Ostrowski, J. Bednarz: Astron. Astrophys. 394, 1141 (2002)

20. A. Panaitescu, P. Kumar: Astrophys. J. 571, 779 (2002) 
21. J. Sakai, T. Nakayama, Y. Kazimura, S. Bulanov: J. Phys. Soc. Japan 69, $2503(2000)$

22. L.O. Silva, R.A. Fonseca, J.W. Tonge, J.M. Dawson, W.B. Mori, M.V. Medvedev: astro-ph 0307500 (2003)

23. B.E. Stern: astro-ph 0301384 (2003)

24. R. Willingale, B. Aschenbach, R.G. Griffiths, S. Sembay, R.S. Warwick, W. Becker, A.F. Abbey, J.M. Bonnet-Bidaud: Astron. Astrophys. 365, L212 (2001)

25. T.Y.B. Yang, Y. Gallant, J. Arons, A.B. Langdon: Phys. Fluids 5, 3369 (1993)

26. T.Y.B. Yang, J. Arons, A.B. Langdon: Phys. Plasmas 1, 3059 (1994) 